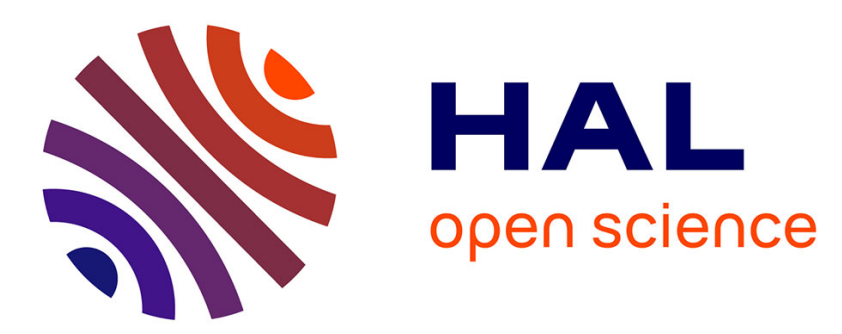

\title{
Numerical investigations of the exergy balance method for aerodynamic performance evaluation
}

Ilias Petropoulos, Christelle Wervaecke, Didier Bailly, Thibaut Derweduwen

\section{To cite this version:}

Ilias Petropoulos, Christelle Wervaecke, Didier Bailly, Thibaut Derweduwen. Numerical investigations of the exergy balance method for aerodynamic performance evaluation. AIAA AVIATION 2019, Jun 2019, DALLAS, United States. 10.2514/6.2019-2926 . hal-02333523

\section{HAL Id: hal-02333523 \\ https://hal.science/hal-02333523}

Submitted on 25 Oct 2019

HAL is a multi-disciplinary open access archive for the deposit and dissemination of scientific research documents, whether they are published or not. The documents may come from teaching and research institutions in France or abroad, or from public or private research centers.
L'archive ouverte pluridisciplinaire $\mathbf{H A L}$, est destinée au dépôt et à la diffusion de documents scientifiques de niveau recherche, publiés ou non, émanant des établissements d'enseignement et de recherche français ou étrangers, des laboratoires publics ou privés. 


\title{
Numerical investigations of the exergy balance method for aerodynamic performance evaluation
}

\author{
Ilias Petropoulos *, Christelle Wervaecke ${ }^{\dagger}$, Didier Bailly ${ }^{\ddagger}$ and Thibaut Derweduwen $\S$ \\ ONERA - The French Aerospace Lab, Meudon, F-92190, France
}

The exergy framework presents an interesting complement to traditional drag extraction methods for the performance evaluation of aerodynamic devices. On the one hand it remains valid for novel configurations for which no thrust/drag distinction is applicable (e.g. Boundary Layer Ingestion), while it can also account for the influence of thermal effects. At the same time, it can provide an additional understanding on the physical phenomena occuring within the flowfield and their interactions, via the destruction of exergy or its transfer from one form to another. The present work aims at providing a further insight into some numerical aspects of the exergy balance approach. A strategy towards the limitation of some of its sensitivities is also presented. This specifically addresses the quantification of spurious generation of anergy, which is directly associated to the spurious generation of entropy in numerical computations.

\section{Nomenclature}

$\begin{array}{ll}p, T & =\text { static pressure and temperature } \\ \rho & =\text { density } \\ u, v, w & =x-, y-, z \text {-component of the velocity vector } \\ e & =\text { mass-specific internal energy } \\ h, s & =\text { mass-specific enthalpy and entropy } \\ \varepsilon & =\text { mass-specific flow exergy } \\ F_{x} & =\text { net streamwise force } \\ k_{\mathrm{eff}} & =\text { effective thermal conductivity } \\ \mathbf{q}_{\mathrm{eff}} & =\text { effective heat flux by conduction on a heating surface } \\ \mathbf{n} & =\text { effective viscous stress tensor } \\ \overline{\bar{\tau}}_{\mathrm{eff}} & =\text { effective dissipation rate per unit volume } \\ \Phi_{\mathrm{eff}} & =\text { thermodynamic or kinematic quantity at the free-stream reference state } \\ (\bullet)_{\infty} & =\text { integration surfaces } \\ S_{o}, S_{w}, S_{p}, S_{b} & =\text { integration volume }\left(\partial \mathcal{V}_{o}=S_{o}\right) \\ \mathcal{V}_{o} & \left.=\text { downstream position of the Trefftz plane (limit of the control volume } S_{o}\right) \\ x_{\mathrm{tp}} & \end{array}$

\section{Introduction}

UMerical methods have an ever-increasing role within the aerodynamic design process of modern aircraft. Due 1 to the increasing complexity of these configurations, solver techniques have significantly evolved over the past decades and nowadays allow the accurate and efficient calculation of a very wide range of fluid flows. Within a research or industrial cycle however, the accurate and efficient evaluation of systems' performance (efficiency, aerodynamic performance coefficients,...) is of an equally crucial importance.

This has lead to multiple families of numerical techniques being developed for the calculation of such performance measures. As regards aerodynamic drag, far-field methods in particular have been proven to be advantageous in terms of sensitivity to numerical error compared to a near-field evaluation. Moreover, such techniques permit the decomposition

\footnotetext{
* Research Scientist, Ph.D., ONERA Department of Aerodynamics, Aeroelasticity and Acoustics, ilias.petropoulos@ onera.fr

${ }^{\dagger}$ Research Scientist, Ph.D., ONERA Department of Aerodynamics, Aeroelasticity and Acoustics, christelle.wervaecke@onera.fr

$\doteqdot$ Research Scientist, Ph.D., ONERA Department of Aerodynamics, Aeroelasticity and Acoustics, didier.bailly@ onera.fr

${ }^{\S}$ Intern, ONERA Department of Aerodynamics, Aeroelasticity and Acoustics, thibaut.derweduwen@onera.fr
} 
of drag into several components depending on the nature of drag sources [1, 2], which in turn can provide precious physical insight to aerodynamic designers.

Design restrictions themselves can arise from regulations, particularly for civil aircraft in terms of pollutant emissions or acoustic signature (e.g. Counter-Rotating Open Rotors). At present however, the design of civil aircraft is largely governed by aerodynamic efficiency. In the search of improved designs, several industrial trends have emerged. These range from being variations of existing concepts, such as ultra-high by-pass ratio (UHBR) dual-stream engines, or more unconventional concepts (Prandtl box-wing planes, distributed propulsion system, blended wing-body etc.).

Another promising concept is the so-called Boundary Layer Ingestion (BLI) [3, 4]. In traditional civil aircraft, engines are deliberately rather segregated from the fuselage to reduce as much as possible the impact of engine integration. BLI designs on the other hand include engines which are highly integrated in the fuselage. This aims at ingesting and directly re-energizing a part of the fuselage's boundary layer, rather than using the engines to separately overpower the momentum defect in the wake. Due to this mechanism, BLI designs can in principle achieve a target net streamwise force with higher efficiency due to a reduced axial/transversal kinetic energy deposition to the free-stream flow.

BLI configurations however entail additional complexities due to the engine integration. The design of the engines themselves is particularly complex as, apart from aerodynamic performance, fan blades must be able to structurally withstand and perform under significant distortion. Furthermore, the aerodynamic performance evaluation of such aircraft is not straightforward because no distinction can be made between thrust and drag, thus making far-field drag extraction methods less interesting on such configurations.

The performance of such novel concepts can be evaluated using more "global" measures, such as done in the power balance method [5]. Another approach for the evaluation of such novel configurations has been developed in ONERA based on the notion of exergy [6-8]. As opposed to the separation of energy into mechanical and thermal (partially convertible to work), the notion of exergy is used to refer to the theoretical work potential of both mechanical and thermal form. This will be discussed in further detail in Section III] It should also be noted that although it may have been largely motivated by it, the exergy analysis is not restricted to the concept of BLI. Furthermore, it is related to the power balance in terms of mechanical energy, but differs in that it can also account for and quantify the influence of thermal effects. It can thus be particularly interesting for the evaluation of aero-thermo-propulsive configurations, such as heat exchanger devices integrated in propulsion systems.

Following the development of a prototype method, this approach has since been applied to a number of academic or industrial configurations [6, 8-10]. A novel implementation of the exergy balance method has been developed to achieve the efficiency required for industrial production and facilitate the development of new functionalities. The present paper itself aims at demonstrating the capabilities of the exergy analysis and at investigating its eventual sensitivities. More specifically, the main focus is put on the identification and quantification of numerical effects, which is essential for the method's industrialization.

\section{Exergy analysis}

The notion of exergy is used to refer to the work potential of a system with respect to its difference from the reference conditions of a heat reservoir. In other words, it represents a measure of the theoretical potential of work which can be extracted from the system by returning it to the reference conditions.

Exergy can naturally appear in many forms (magnetic, chemical, physical,...), similarly to energy. Without loss of generality, the focus is primarily put on physical and chemical exergy for aerodynamics applications. By neglecting gravitational potential exergy and also considering a perfect gas hypothesis (thus neglecting chemical exergy), the definition of exergy can be written:

$$
\varepsilon \triangleq h_{i}-h_{i, \infty}-T_{\infty}\left(s-s_{\infty}\right)
$$

where $s$ denotes entropy and $h_{i}$ denotes specific total enthalpy $\left(h_{i}=h+\frac{1}{2} V^{2}\right)$. For external aerodynamics applications, the reference conditions are usually taken as the atmosphere free-stream flow.

\section{A. Formulation of the exergy balance}

The method aims at evaluating the time-averaged changes in exergy. It is thus oriented towards the post-processing of Reynolds-averaged Navier-Stokes (RANS) solutions. Based on the momentum relation, mass conservation and thermodynamic relations, a general exergy balance equation is obtained [6, 7]:

$$
\dot{\mathcal{E}}_{\text {prop }}+\dot{\mathcal{E}}_{q}=W \dot{\Gamma}+\dot{\mathcal{E}}_{m}+\dot{\mathcal{E}}_{t h}+\dot{\mathcal{A}}_{\Phi}+\dot{\mathcal{A}}_{\nabla T}+\dot{\mathcal{A}}_{w}
$$




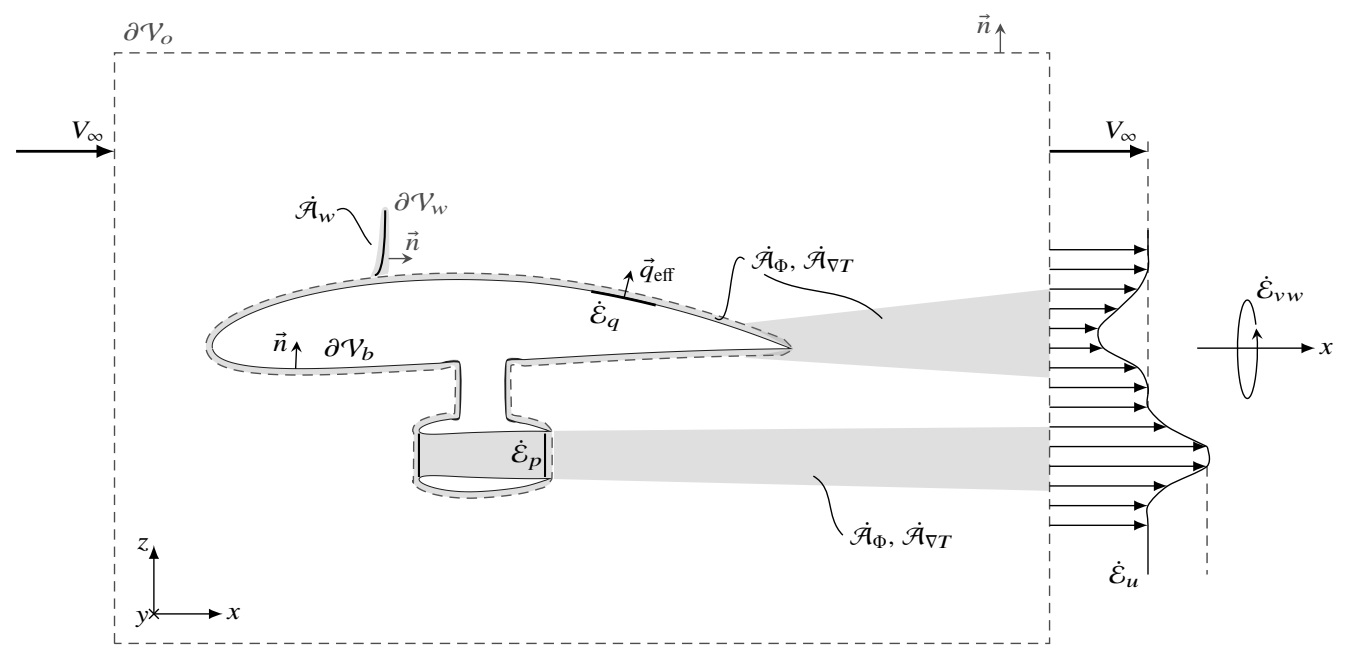

Fig. 1 Illustration of integration surfaces in the $x z$-plane of a three-dimensional volume. It is $S_{A}=S_{b} \cup S_{p}$ and $\partial \mathcal{V}_{o}=S_{o}$.

The balance of Eq. (2) accounts for aerodynamic, propulsion system, as well as thermal phenomena occurring within a control volume. It can thus be used for the performance evaluation of aero-thermo-propulsive systems through exergy (i.e. work potential) loss or transformation from one form to another.

The term $W \dot{\Gamma}$ is written:

$$
W \dot{\Gamma}=V_{\infty} \int_{S_{o}}\left[\rho u(\mathbf{V} \cdot \mathbf{n})+\left(p-p_{\infty}\right) n_{x}\right] d S=-F_{x} V_{\infty}
$$

where $\Gamma$ is the weight specific aircraft energy height, i.e. the sum of its potential and kinetic energy. This term is obtained from the momentum relation and is equal to the power (i.e. the rate of work) of the resultant net streamwise force, calculated in the far-field. As such, it should be underlined that the above expression provides no distinction between thrust and drag. More precisely, this term represents the potential utilized by the aircraft to maintain a steady path.

The two terms $\dot{\mathcal{E}}_{\text {prop }}+\dot{\mathcal{E}}_{q}$ on the left-hand side of Eq. [2] represent the inflow of exergy to the system through propulsive, non-adiabatic or permeable surfaces. $\dot{\mathcal{E}}_{\text {prop }}$ represents the flow of exergy via the propulsion system:

$$
\dot{\mathcal{E}}_{\text {prop }}=-\int_{S_{p}} \rho \delta h_{i}(\mathbf{V} \cdot \mathbf{n}) d S+T_{\infty} \int_{S_{p}} \rho \delta s(\mathbf{V} \cdot \mathbf{n}) d S
$$

where $\delta(\bullet)=(\bullet)-(\bullet)_{\infty}$ and $S_{p}$ is the surface enclosing the propulsion system. The term $\dot{\mathcal{E}}_{q}$ represents the flow of exergy via thermal conduction:

$$
\dot{\mathcal{E}}_{q}=-\int_{S_{b}}\left(\mathbf{q}_{\mathrm{eff}} \cdot \mathbf{n}\right) d S+\int_{S_{b}} \frac{T_{\infty}}{T_{w}}\left(\mathbf{q}_{\mathrm{eff}} \cdot \mathbf{n}\right) d S
$$

where $T_{w}$ denotes the local temperature on the body walls and $\mathbf{q}_{\text {eff }}$ denotes the effective heat flux by conduction $\left(\mathbf{q}_{\mathrm{eff}}=-k_{\mathrm{eff}} \nabla \mathbf{T}\right)$.

The right-hand side terms of Eq. (2) represent the decomposition of exergy into different forms within the control surface $S_{o}$. The term $\dot{\mathcal{E}}_{m}$ represents the rate of mechanical exergy outflow:

$$
\dot{\mathcal{E}}_{m}=\int_{S_{o}} \frac{1}{2} \rho u^{2}(\mathbf{V} \cdot \mathbf{n}) d S+\int_{S_{o}} \frac{1}{2} \rho\left(v^{2}+w^{2}\right)(\mathbf{V} \cdot \mathbf{n}) d S+\int_{S_{o}}\left(p-p_{\infty}\right)\left[\left(\mathbf{V}-\mathbf{V}_{\infty}\right) \cdot \mathbf{n}\right] d S
$$

The two first integrals in Eq. (6) are respectively the streamwise and transversal kinetic exergy deposition rate. The first $\left(\dot{\mathcal{E}}_{u}\right)$ is related to jets or the wake, while the second $\left(\dot{\mathcal{E}}_{v w}\right)$ is related to transversal kinetic energy such as the lift-induced vortices associated with induced drag. The last term $\left(\dot{\mathcal{E}}_{p}\right)$ is a boundary pressure-work rate related to pressure and velocity differences on the surface $S_{o}$ with respect to the reference state. 
The rate of thermal exergy outflow $\dot{\mathcal{E}}_{t h}$ is given by:

$$
\dot{\mathcal{E}}_{t h}=\int_{S_{o}} \rho \delta e(\mathbf{V} \cdot \mathbf{n}) d S+\int_{S_{o}} p_{\infty}(\mathbf{V} \cdot \mathbf{n}) d S-T_{\infty} \int_{S_{o}} \rho \delta s(\mathbf{V} \cdot \mathbf{n}) d S
$$

and is decomposed in three parts respectively representing the rate of thermal energy outflow, the rate of isobaric surroundings work and the outflow rate of anergy (def. below).

The last three terms in Eq. (2) represent the generation of anergy within the control volume. This term is used to refer to the loss of work potential through irreversible processes. In the exergy framework, anergy mechanisms are often also referred to as mechanisms of loss or destruction of exergy.

The rate of anergy generation by viscous dissipation is given by:

$$
\dot{\mathcal{A}}_{\Phi}=\int_{\mathcal{V}_{o}} \frac{T_{\infty}}{T} \Phi_{\text {eff }} d \mathcal{V}
$$

where $\Phi_{\text {eff }}=\left(\overline{\bar{\tau}}_{\text {eff }} \cdot \nabla\right) \cdot \mathbf{V}$ is the effective dissipation rate per unit volume. The viscous mechanisms contained in $\dot{\mathcal{A}}_{\Phi}$ transform differences in kinetic energy to thermal energy, thus leading the system towards a mechanical equilibrium with homogeneous velocity/pressure field. The term $\dot{\mathcal{A}}_{\nabla T}$ is the rate of anergy generation by thermal conduction:

$$
\dot{\mathcal{A}}_{\nabla T}=\int_{\mathcal{V}_{o}} \frac{T_{\infty}}{T^{2}} k_{\mathrm{eff}}(\nabla \mathbf{T})^{2} d \mathcal{V}
$$

and represents the loss of work potential through thermal diffusion due to temperature differences. Finally, $\dot{\mathcal{A}}_{w}$ is the rate of anergy generation by shockwaves:

$$
\dot{\mathcal{A}}_{w}=T_{\infty} \int_{\partial V_{w}} \rho \delta s(\mathbf{V} \cdot \mathbf{n}) d S
$$

where the normal $\mathbf{n}$ is shown in Fig. 11 The anergy terms of Eqs. (88)-(10) are positive and can be collectively referred to as the total rate of anergy generation $\left(\dot{\mathcal{A}}_{\text {tot }}=\dot{\mathcal{A}}_{\Phi}+\dot{\mathcal{A}}_{\nabla T}+\dot{\mathcal{A}}_{w}\right)$.

\section{B. Physical Interpretation}

Eq. (2) represents the balance within the control volume between: the inflow of exergy into the system on the left-hand side and its eventual transformation into work, its deposition to the free-stream flow or the loss of work potential on the right-hand side. In a powered configuration, the inflow of exergy (left-hand side of Eq. (2)) is provided to produce a net streamwise thrust (i.e. $F_{x}$ ). This exergy is however always supplied in excess, as part of it is transferred to the free-stream flow in the form of mechanical $\left(\dot{\mathcal{E}}_{m}\right)$ or thermal $\left(\dot{\mathcal{E}}_{t h}\right)$ exergy, whereas another part of this work potential is lost through irreversible processes $\left(\dot{\mathcal{A}}_{\nabla T}, \dot{\mathcal{A}}_{\Phi}, \dot{\mathcal{A}}_{w}\right)$. It should be noted that, even though the mechanical and thermal exergy outflow terms (i.e. $\dot{\mathcal{E}}_{m}, \dot{\mathcal{E}}_{t h}$ ) do not represent exergy losses, they still represent work which is not given for the required streamwise force. A good design would thus achieve a target streamwise force with a minimum kinetic/thermal exergy deposition to the free-stream and a minimum loss of work potential. Equivalently, this means that a good design requires a minimum inflow of exergy $\left(\dot{\mathcal{E}}_{\text {prop }}, \dot{\mathcal{E}}_{q}\right)$ to achieve the target streamwise force.

Unlike traditional aerodynamic force extraction methods, the exergy decomposition is by definition dependent on the volume $\mathcal{V}_{o}$ (i.e. on $\partial \mathcal{V}_{o}=S_{o}$ ). Contrary to aerodynamic forces, exergy changes from one form to another in the proximity of aerodynamic bodies and further downstream. This is because thermal mixing and viscous mechanisms lead to the gradual (physical and numerical) dissipation of exergy as the system tends towards equilibrium with the reference state. As shown in Fig. 1. the control volume is limited by a downstream position of the Trefftz plane. A variation of this downstream limit can give an insight into flow physics via the loss of exergy, or its transfer from one form to another downstream of the aerodynamic body. This mechanism is illustrated in Fig. 2. as a downstream decrease of mechanical/thermal exergy components and an increase of anergy terms.

For practical purposes, the terms of Eq. (2) are nondimensionalized by:

$$
(\bullet)=(\bullet) / \frac{1}{2} \rho_{\infty} V_{\infty}^{3} A_{\text {ref }}
$$

where $A_{\text {ref }}$ denotes a reference surface. The non-dimensional values of the exergy terms obtained via Eq. (11) are thus given in power counts (p.c.), in analogy to traditional drag counts. 


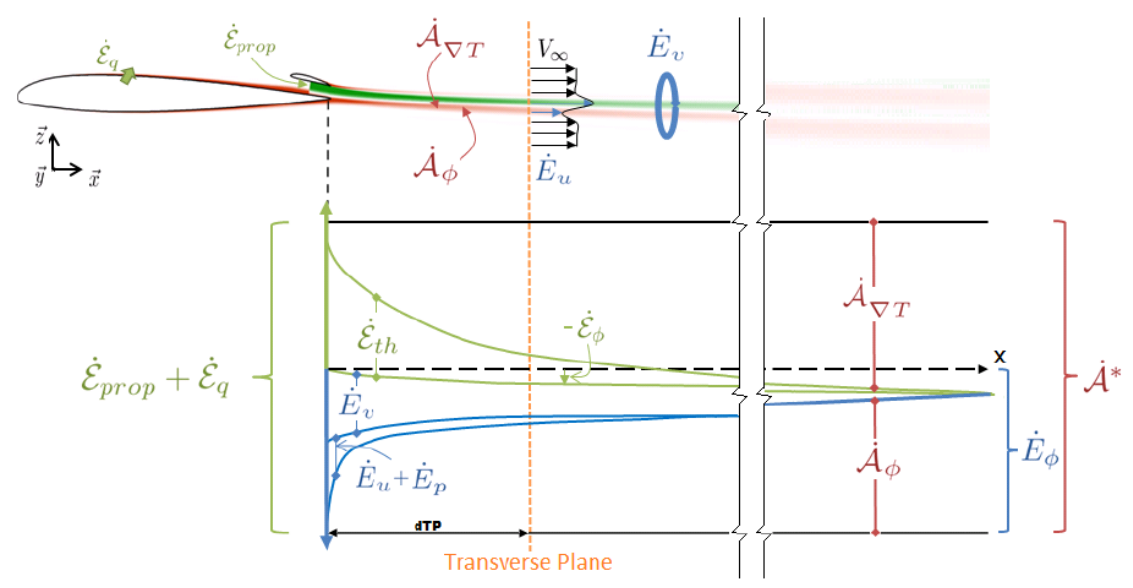

Fig. 2 Variation of exergy balance terms for varying position of the Trefftz plane. The variation of the term $\dot{\mathcal{A}} w$ is not shown. $\dot{E}_{\Phi}$ and $\dot{\mathcal{E}}_{\Phi}$ are respectively the rate of thermal energy and the rate of thermal exergy generation by viscous dissipation, $\dot{\mathcal{A}}^{*}=\dot{\mathcal{E}}_{m}+\dot{\mathcal{E}}_{t h}+\dot{\mathcal{A}}_{t o t}$ and the notations $\dot{E}_{u}, \dot{E}_{v}, \dot{E}_{p}$ respectively correspond to the components of $\dot{\mathcal{E}}_{m}$ (see Eq. (6)). Figure taken from Ref. [6].

\section{Post-processing method}

As already discussed in Section II] post-processing codes are required to keep up with the rapid evolutions of CFD solvers in terms of flexibility and computational efficiency in modern architectures. To attain this objective, the implementation of the exergy balance method presented in Section III.A is coupled with Cassiopé 周 a partly open-source library of functions developed at ONERA for the pre- and post-processing of CFD solutions [11].

The exergy analysis is contained in a separate post-processing module called FFX (Far-Field Exergy), developed at ONERA. CFD solutions including computational mesh and connectivity information are based on the CGNS standard (CFD General Notation System) [12]. The interface is implemented in Python, offering significant flexibility (scripting, co-processing, coupling with other modules,...). Internal computations are handled in Python/C++, allowing the combination of flexibility and computational performance. The current version of the software allows the exergy post-processing of CFD solutions on structured or unstructured grids, with the flow solution being located either at cell centers or at vertices. It can also handle several treatments and boundary conditions relative to aeronautic applications such as Actuator Disk models, Fan/OGV modelization with Body-Force terms, heat exchanger boundary conditions etc. Some results of applications of $F F X$ to industrial cases can be found in Refs. [9, 10, 13]. For the convergence sensitivity study presented in Section IV.A the computation of the exergy decomposition with $F F X$ was performed by memory coupling with the solver and on-the-fly post-processing during the CFD computation.

The presented results of a far-field decomposition of the drag force were performed with the $F F D$ (Far-Field Drag) software developed at ONERA. This is based on an implementation of the far-field drag decomposition method of ONERA [1, 2] on a similar software architecture with $F F X$.

\section{Numerical applications}

In the following, numerical investigations of the exergy balance approach are performed by application to a series of aerodynamics computations. These include sensitivity analyses (convergence of the computation, mesh refinement), as well as investigations aiming at further increasing the precision of the formulation (definition of integration volumes using physical criteria). The RANS solutions presented below were computed with the ONERA-Airbus-SAFRAN elsA CFD solver [14].

\section{A. Sensitivity to convergence level and grid density}

The present section presents a series of sensitivity analyses of the exergy balance method with respect to the precision of the RANS CFD solution. This is regarded from two separate aspects: the level of convergence and the grid density.

\footnotetext{
*http://elsa.onera.fr/Cassiopee/
} 

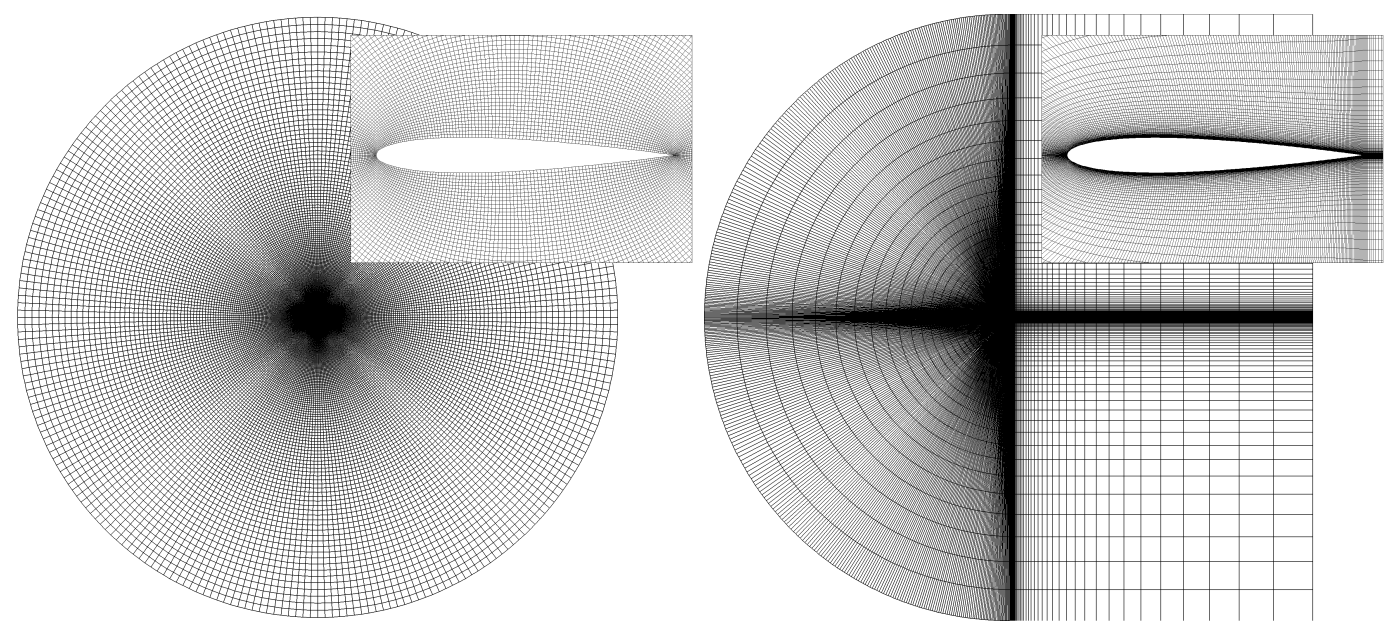

Fig. 3 View of the $256 \times 256$ O-type Euler grid (left) and the $512 \times 128$ C-type RANS grid (right) for the NACA0012 airfoil computations.
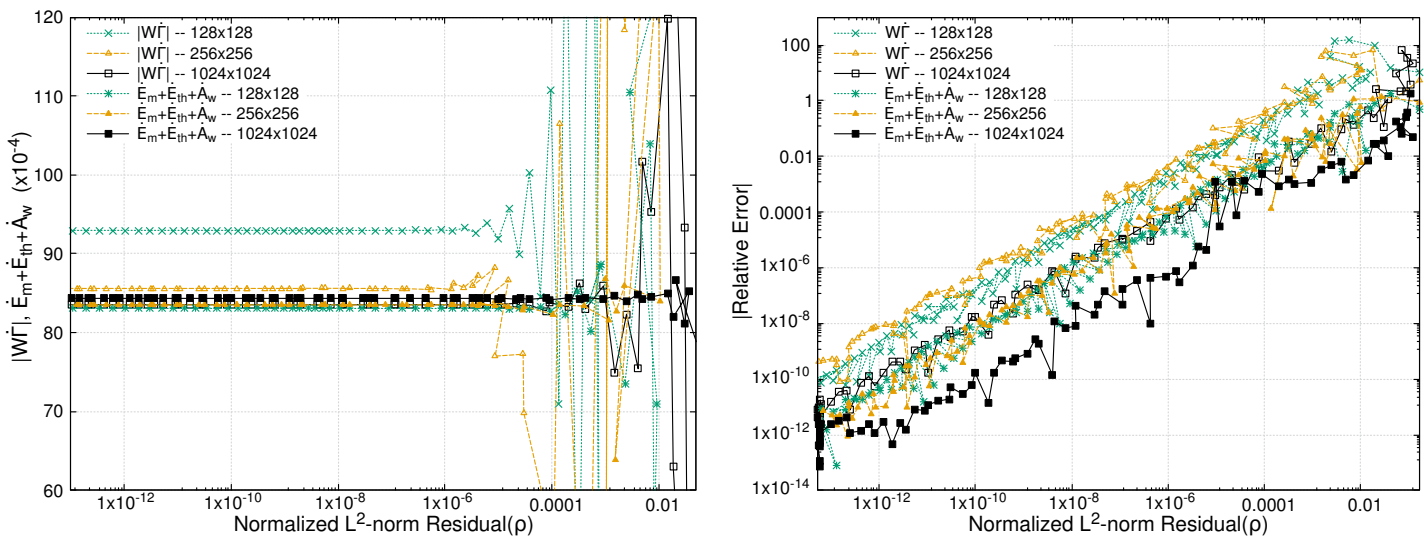

Fig. 4 Convergence of the exergy balance with respect to the reduction of the residual of the mass-conservation equation for the inviscid NACA0012 case at $M_{\infty}=0.8$ on different grids.

The first case is a NACA0012 airfoil in an inviscid transonic flow $\left(M_{\infty}=0.8, \alpha=0^{\circ}, p_{\infty}=101325 \mathrm{~Pa}, T_{\infty}=300 \mathrm{~K}\right)$. Computations were performed with the Jameson spatial discretization scheme $\left(k_{2}=1 / 2, k_{4}=1 / 64\right)$. The case is solved on a series of high-quality O-type analytical Euler grids composed of $128 \times 128,256 \times 256$ and $1024 \times 1024$ cells (denoted as $n_{i} \times n_{j}$ ), originating from the study presented in Ref. [15]. The far-field extent is at approximately $150 c$, where $c$ is the airfoil chord (see Fig. 3). It is reminded that for solutions of the Euler equations, viscous and heat transfer terms $\left(\dot{\mathcal{E}}_{q}, \dot{\mathcal{A}}_{\nabla T}, \dot{\mathcal{A}}_{\Phi}\right)$ are zero. In this case, the resultant net streamwise force acting on the airfoil is equal to drag since no thrust is provided on non-powered configurations.

Fig. 4 presents the evolution of the exergy terms with respect to the level of convergence of the residual of the mass conservation equation. The downstream limit of the control surface $S_{o}$ was taken at $x_{\mathrm{tp}}=2.0$. Errors due to numerical approximation, especially on coarse meshes, can lead to the exergy balance of Eq. (2) not being exactly verified on the discrete level. Since each term is calculated directly and independently from the others, the difference between $W \dot{\Gamma}$ and the sum of the other terms can also be regarded as a measure of accuracy. Furthermore, the mass-conservation residual is a quite representative measure of convergence, as conservation in the flow field can be more important for terms calculated in the far-field than near-field ones. It is shown that $W \dot{\Gamma}$ is more sensitive than the other terms at early stages of convergence. The convergence of all terms towards the values of the converged solution is almost linear with respect to the reduction of the mass-conservation residual on this case. The overall accuracy of the numerical method is however satisfactory, the value of $W \dot{\Gamma}$ being within below $1 \%$ of error with respect to the reference values after a residual reduction of approximately five orders of magnitude on the two coarser grids and three orders of magnitude on the fine grid. As for mechanical exergy and wave anergy terms, a reduction by three to four orders of magnitude has 


\begin{tabular}{cccccccccccc}
\hline & Grid & $C_{D, n f}$ & $C_{D, f f}$ & $C_{D, s p}$ & $W \dot{\Gamma}$ & $\dot{\mathcal{E}}_{u}$ & $\dot{\mathcal{E}}_{v w}$ & $\dot{\mathcal{E}}_{p}$ & $\dot{\mathcal{E}}_{t h}$ & $\dot{\mathcal{A}}_{w}$ & Residual \\
\hline$M_{\infty}=0.4$ & $n_{i}=128$ & 5.93 & 0.00 & 5.93 & -6.01 & 0.65 & 0.50 & -1.24 & 0.10 & 0.00 & 6.00 \\
& $n_{i}=256$ & 1.49 & 0.00 & 1.49 & -1.50 & 0.61 & 0.51 & -1.21 & 0.09 & 0.00 & 1.50 \\
& $n_{i}=1024$ & 0.09 & 0.00 & 0.09 & -0.09 & 0.60 & 0.50 & -1.20 & 0.10 & 0.00 & 0.09 \\
$M_{\infty}=0.8$ & $n_{i}=128$ & 92.84 & 84.74 & 8.09 & -92.96 & 5.30 & 1.29 & -8.22 & 2.33 & 82.37 & 9.89 \\
& $n_{i}=256$ & 85.60 & 83.75 & 1.85 & -85.61 & 5.07 & 1.31 & -8.13 & 2.30 & 82.97 & 2.08 \\
& $n_{i}=1024$ & 83.53 & 83.55 & -0.02 & -83.53 & 5.03 & 1.31 & -8.12 & 2.30 & 83.83 & -0.82 \\
\hline
\end{tabular}

Table 1 Far-field drag and exergy decomposition for the inviscid NACA0012 case at $x_{\mathrm{tp}}=2.0$. Drag and exergy terms are presented in drag and power counts respectively $\left(\times 10^{-4}\right)$.

been found to suffice to obtain the same accuracy even on the coarse grid.

Values of the exergy balance terms for the converged computations on the three grids are presented in Table 1 . The exergy decomposition is also shown for a subsonic inviscid computation of the NACA0012 airfoil at $M_{\infty}=0.4$, $\alpha=0^{\circ}, p_{\infty}=101325 \mathrm{~Pa}, T_{\infty}=300 \mathrm{~K}$. The artificial dissipation coefficients for the Jameson scheme were taken $k_{2}=0$, $k_{4}=1 / 64$ for this case. It is reminded that for the subsonic regime, the exact inviscid solution is known a priori to correspond to zero drag. On the other hand, drag is solely composed of the wave drag far-field component for the transonic case.

It can be remarked that the exergy balance residual for the subsonic regime is almost exclusively associated to the $W \dot{\Gamma}$ value, the sum of mechanical and thermal exergy terms $\left(\dot{\mathcal{E}}_{u}, \dot{\mathcal{E}}_{v w}, \dot{\mathcal{E}}_{p}, \dot{\mathcal{E}}_{t h}\right)$ being very close to zero even on the coarsest grid. A far-field drag decomposition is also presented. The values given for spurious drag $C_{D, s p}$ correspond to the irreversible spurious drag component, its reversible counterpart being of a lower order of magnitude for these computations [16]. Results show that a far-field drag computation is more accurate than a near-field evaluation and converges faster with grid refinement. In terms of nondimensionalized absolute value, $W \dot{\Gamma}$ is found to be close to that of the near-field drag component. With the exception of $W \dot{\Gamma}$, exergy terms are not found to be very sensible to the grid in this case.

The same study is then performed for RANS solutions of the NACA0012 airfoil in subsonic $\left(M_{\infty}=0.3, \alpha=0^{\circ}\right.$, $\left.R e=310^{6}\right)$ and transonic regime $\left(M_{\infty}=0.8, \alpha=0^{\circ}, R e=810^{6}\right)$. The artificial dissipation parameters for the Jameson scheme were set to $k_{2}=0.0, k_{4}=1 / 128$ for the $M_{\infty}=0.3$ case and to $k_{2}=0.5, k_{4}=1 / 64$ for the $M_{\infty}=0.8$ case. Computations were performed with the Spalart-Allmaras turbulence model. The case is investigated on three different C-type grids of varying density, composed of $256 \times 64,512 \times 128$ and $1024 \times 256$ cells. A view of the medium grid is shown in Fig. 3 .

The evolution of the terms with respect to the convergence of the density residual is shown in Fig.5 In this viscous case, the early convergence of the exergy terms is faster with respect to the transonic Euler computation. An error threshold of $1 \%$ with respect to the reference value on all terms is reached after a reduction of the density residual by approximately five orders of magnitude. Once again, the convergence of the term $W \dot{\Gamma}$ is shown to be slower compared to the convergence of the other terms of the exergy decomposition. Also, contrary to the Euler case, the exergy balance terms are found to converge faster on the coarser grid.

\begin{tabular}{ccccccccccccc}
\hline & Grid $n_{i}$ & $C_{D, n f}$ & $C_{D, w}$ & $C_{D, f f}$ & $C_{D, s p}$ & $W \dot{\Gamma}$ & $\dot{\mathcal{E}}_{m}$ & $\dot{\mathcal{E}}_{t h}$ & $\dot{\mathcal{A}}_{\Phi}$ & $\dot{\mathcal{A}}_{\nabla T}$ & $\dot{\mathcal{A}}_{w}$ & Residual \\
\hline$M_{\infty}=0.3$ & 256 & 91.17 & 0.00 & 90.84 & 0.32 & -91.29 & 4.67 & 0.19 & 82.68 & 1.01 & 0.00 & 2.75 \\
& 512 & 90.89 & 0.00 & 90.86 & 0.03 & -90.88 & 4.25 & 0.17 & 84.59 & 1.06 & 0.00 & 0.81 \\
& 1024 & 90.73 & 0.00 & 90.74 & 0.00 & -90.72 & 4.11 & 0.17 & 85.02 & 1.07 & 0.00 & 0.36 \\
$M_{\infty}=0.8$ & 256 & 163.07 & 59.36 & 160.12 & 2.94 & -162.70 & 3.52 & 3.26 & 79.93 & 9.30 & 56.04 & 10.65 \\
& 512 & 158.38 & 60.15 & 157.92 & 0.45 & -158.32 & 3.03 & 3.02 & 80.84 & 9.52 & 59.33 & 2.59 \\
& 1024 & 157.32 & 60.64 & 157.26 & 0.05 & -157.29 & 2.93 & 2.89 & 80.84 & 9.52 & 60.69 & 0.42 \\
\hline
\end{tabular}

Table 2 Far-field drag and exergy decomposition for the viscous NACA0012 case at $x_{\text {tp }}=2.0$. Drag and exergy terms are presented in drag and power counts respectively $\left(\times 10^{-4}\right)$.

Table 2 shows values of exergy balance terms on the converged computations. The rate of anergy generation due to shockwaves is close to the value of the far-field wave drag component $C_{D, w}$. The values of these two terms can be 

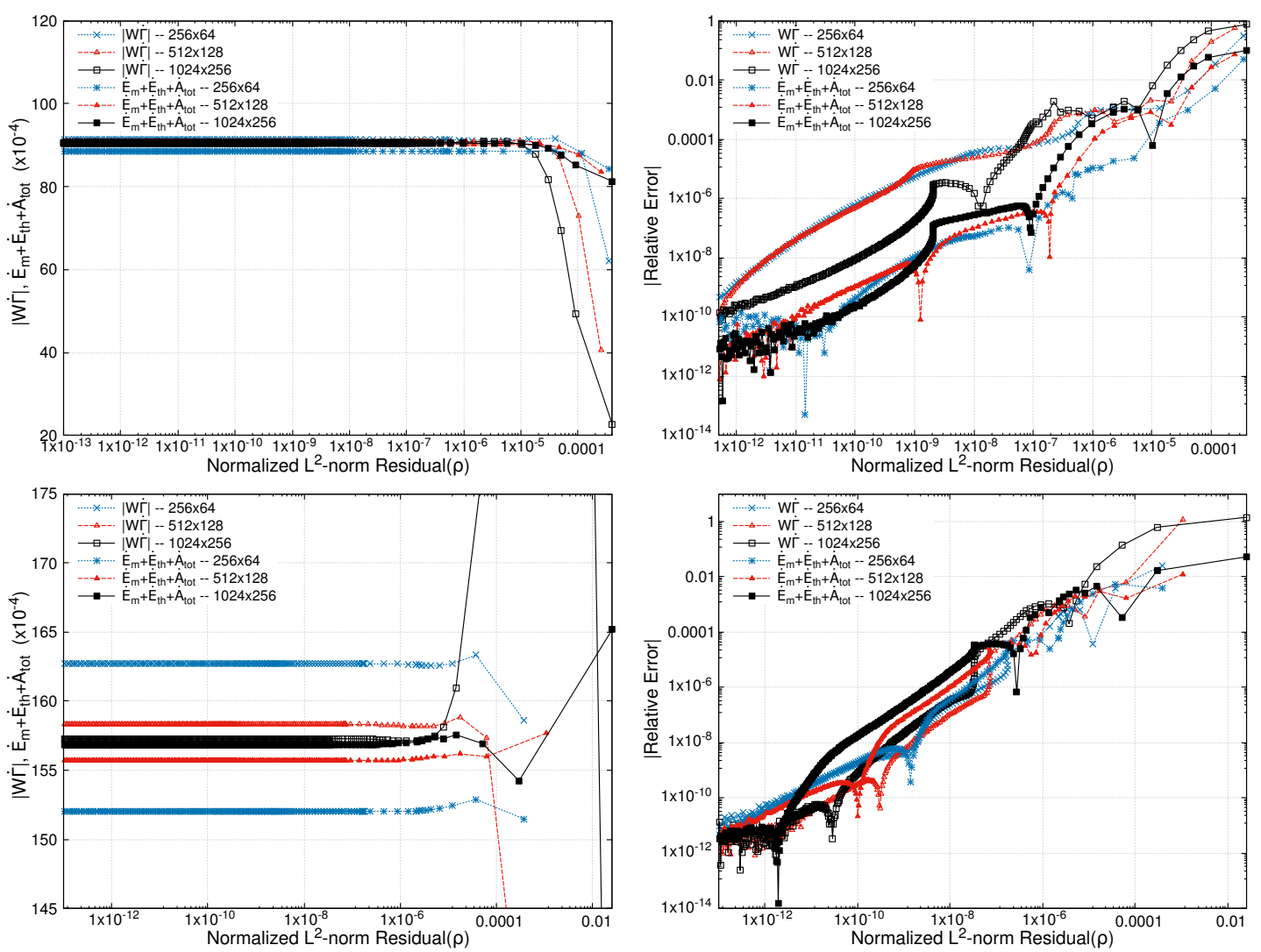

Fig. 5 Convergence of the exergy balance with respect to the reduction of the residual of the mass-conservation equation for the viscous NACA0012 case at $M_{\infty}=0.3$ (top) and at $M_{\infty}=0.8$ (bottom) on different grids.

comparable under an appropriate nondimensionalization, even though the terms themselves have a different physical content. This however cannot be straightforwardly generalized to any regime, e.g. to high-Mach-number flows. As regards the convergence of drag, a far-field drag computation is again shown to be superior to a near-field evaluation. The accuracy of the exergy balance (i.e. the discrete exergy balance residual) converges with grid refinement. Moreover, the difference between the residual of the exergy balance compared to spurious drag is higher than for the inviscid case.

Fig. 6 6 presents the evolution of some terms of the exergy balance for variation of the downstream limit of the control volume. These variations show little sensitivity to mesh density on this case. As already discussed in Section III.B. such graphs allow to monitor the evolution of exergy terms downstream of the aerodynamic body. More specifically, they portray the reduction of kinetic/thermal exergy in the wake and the increase of rate of anergy generation due to irreversible processes (viscous dissipation, thermal conduction). More localized physical insight can be obtained by visualizations of the volumic field of $\dot{\mathcal{A}}_{\Phi}, \dot{\mathcal{A}}_{\nabla T}$ (see Fig. 6). These can be used to identify flow regions where losses of exergy (i.e. work potential) are dominant, either due to numerical discretization error or due to physical phenomena. In the NACA0012 airfoil case, loss of exergy is concentrated in the shockwave, boundary layer and the airfoil wake. Spurious production of anergy is also observed near the airfoil leading edge (cf. discussion of Section IV.B].

As a following step, the wing-body configuration of the Common Research Model (CRM) is considered. This is in order to evaluate the extensibility of the observations on airfoil cases to a more complex and realistic three-dimensional aeronautic configuration. Exergy analyses have already been presented on this configuration during the development of the formulation presented in Section [III.A [6, 8]. The original version of the grids corresponds to the multi-block unified baseline grids of the 5th AIAA CFD Drag-Prediction Workshop [17]. The current computations were performed on a series of grids adapted to the experimentally measured wing twist at the design point [18]. The number of cells for the $\mathrm{L} 2^{\prime} / \mathrm{L} 3^{\prime} / \mathrm{L}^{\prime}$ grid levels is $2,156,544 / 5,111,808 / 17,252,352$ respectively $\left(Y^{+}=1.33 / 1.00 / 0.67\right)$. The conditions at the aerodynamic design point correspond to Mach number $M_{\infty}=0.85, C_{L}=0.5$ and $R e=510^{6}$. The aerodynamic force coefficients for the three grid levels are presented in Table 3. 

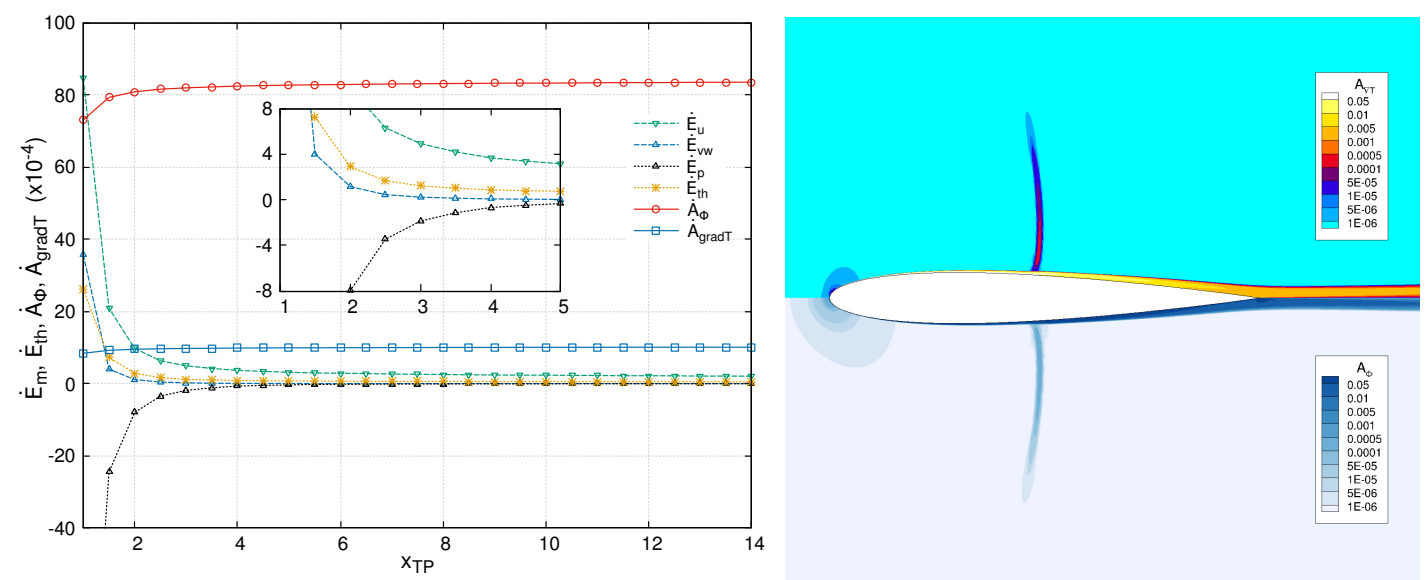

Fig. 6 Left: Evolution of exergy ourflow and anergy generation terms for variation of the downstream limit $x_{\text {tp }}$. Right: field of rate of anergy generation due to viscous dissipation and thermal conduction. NACA0012 solution on the $1024 \times 256$ grid at $M_{\infty}=0.8$.

\begin{tabular}{|c|c|c|c|c|c|c|c|c|c|c|}
\hline \multirow[b]{2}{*}{ Grid level } & \multirow[b]{2}{*}{$C_{L}$} & \multirow[b]{2}{*}{$C_{M}$} & \multicolumn{3}{|c|}{ Near-Field Drag } & \multicolumn{5}{|c|}{ Far-Field Drag } \\
\hline & & & $C_{D, p}$ & $C_{D, f}$ & $C_{D, n f}$ & $C_{D, v p}$ & $C_{D, w}$ & $C_{D, i}$ & $C_{D, f f}$ & $C_{D, s p}$ \\
\hline $\mathrm{L}^{\prime \prime}$ & 0.4997 & -0.0868 & 144.71 & 113.62 & 258.32 & 43.57 & 5.91 & 93.66 & 256.76 & 1.56 \\
\hline L3' & 0.4999 & -0.08 & 142.36 & 114.00 & 256 & 42.41 & 5.83 & 93.43 & 255.66 & 0.69 \\
\hline $\mathrm{L} 4^{\prime}$ & 0.4995 & -0.0896 & 140.23 & 114.47 & 254.71 & 41.35 & 5.77 & 92.92 & 254.51 & 0.19 \\
\hline
\end{tabular}

Table 3 Far-field drag decomposition for the CRM configuration. Drag coefficients are presented in drag counts $\left(\times 10^{-4}\right)$.
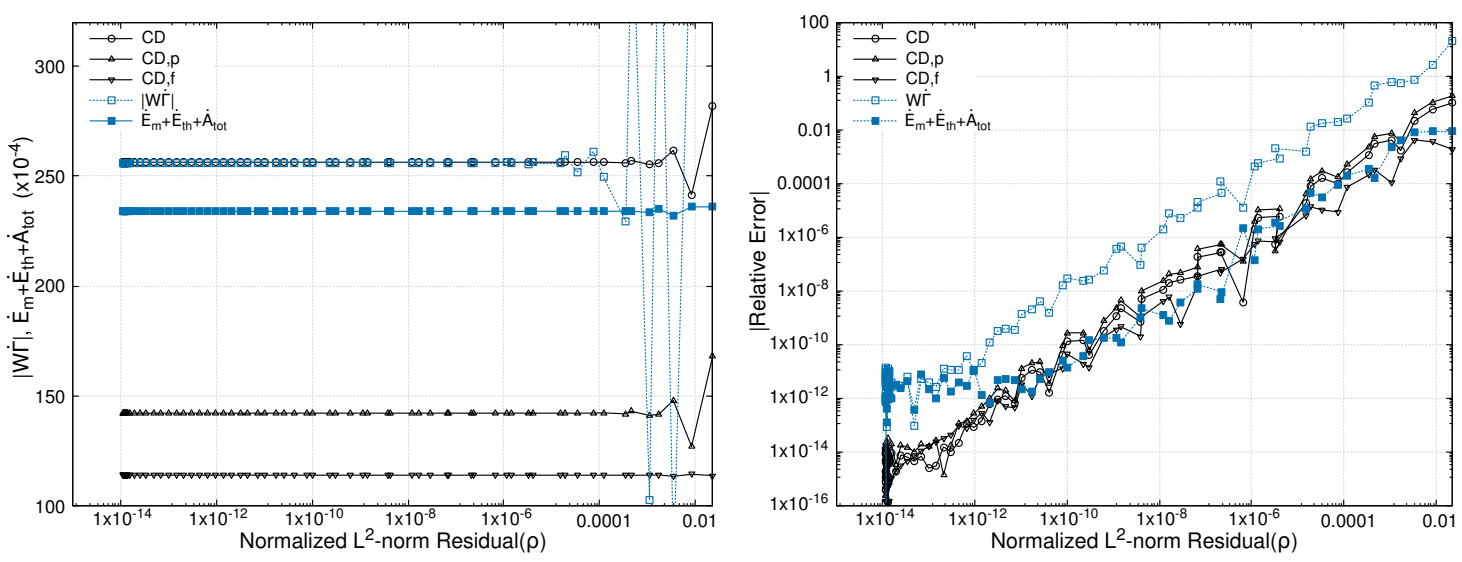

Fig. 7 Convergence of near-field drag coefficients and exergy balance with respect to the reduction of the residual of the mass-conservation equation for the CRM case on the $\mathrm{L3}^{\prime}$ grid.

The convergence of near-field drag components (pressure and friction drag) and the exergy decomposition is shown in Fig. 7 for the L3' grid. The downstream limit of the control volume has been taken at $x_{\mathrm{tp}}=90$, downstream of the fuselage trailing edge. The value of $W \dot{\Gamma}$ is more sensitive at the early stages of convergence. The error with respect to the converged value of $W \dot{\Gamma}$ is less than $1 \%$ after a reduction of the mass-conservation equation residual by five orders of magnitude. On the other hand, a reduction by three orders of magnitude is sufficient to obtain the same accuracy for the near-field drag components and the other terms of the exergy decomposition. In terms of converged absolute values, $W \dot{\Gamma}$ is within 0.2 counts of the near-field drag coefficient. The convergence of the friction drag component is shown to be faster than that of pressure drag. Still, the difference between $W \dot{\Gamma}$ against the exergy deposition and anergy 


\begin{tabular}{|c|c|c|c|c|c|c|c|c|c|c|c|}
\hline \multirow[b]{2}{*}{ Grid level } & \multicolumn{8}{|c|}{ Individual terms } & \multicolumn{3}{|c|}{ Collective terms } \\
\hline & $W \dot{\Gamma}$ & $\dot{\mathcal{E}}_{u}$ & $\dot{\mathcal{E}}_{v w}$ & $\dot{\mathcal{E}}_{p}$ & $\dot{\mathcal{E}}_{t h}$ & $\dot{\mathcal{A}}_{w}$ & $\dot{\mathcal{A}}_{\Phi}$ & $\dot{\mathcal{A}}_{\nabla T}$ & $\dot{\mathcal{E}}_{m}$ & $\dot{\mathcal{A}}_{\text {tot }}$ & Residual \\
\hline $\mathrm{L} 2^{\prime}$ & -257.97 & 6.40 & 83.43 & -1.98 & 1.84 & 4.37 & 122.72 & 11.96 & 87.86 & 139.05 & 29.22 \\
\hline $\mathrm{L}^{\prime}$ & -256.18 & 6.25 & 84.82 & -1.95 & 1.82 & 4.73 & 125.86 & 12.51 & 89.13 & 143.10 & 22.14 \\
\hline $\mathrm{L} 4^{\prime}$ & -254.63 & 6.42 & 86.34 & -1.99 & 1.86 & 5.22 & 128.89 & 13.03 & 90.77 & 147.14 & 14.86 \\
\hline
\end{tabular}

Table 4 Exergy decomposition on different grid levels for the CRM case at $x_{\text {tp }}=90$.
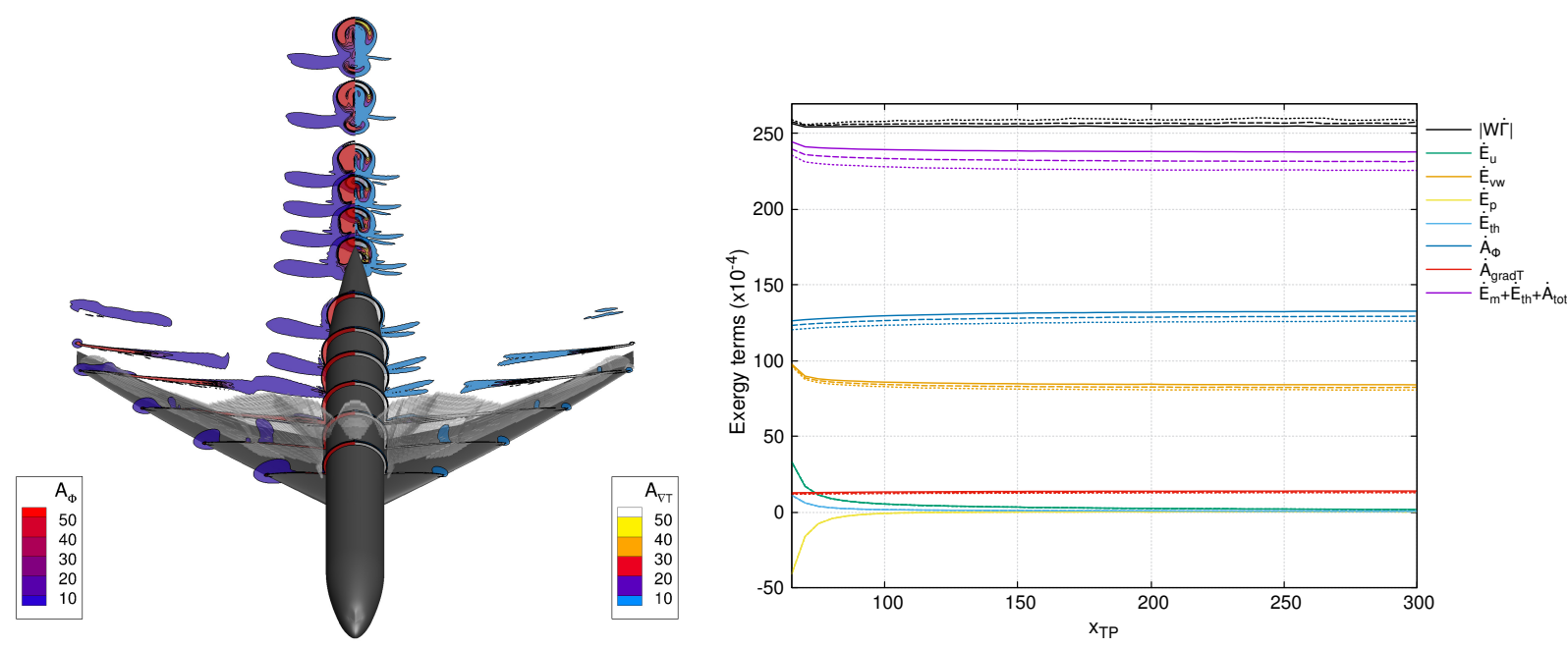

Fig. 8 Left: contours of rate of anergy generation by viscous dissipation and by thermal conduction at various $\mathrm{x}$-positions on the $\mathrm{L3}^{\prime}$ grid. The three-dimensional shockwave integration volume is also shaded. Only half of the shown configuration was computed. Right: evolution of the exergy decomposition terms by variation of the downstream limit of the control volume on the three grids, L2' (dotted lines), L3' (dashed lines) and L4' (solid lines).

generation in the flow field (i.e. the discrete exergy balance residual) remains significant. It is however important to note that this residual is not an effect of insufficient convergence of the CFD computation.

Table 4 shows the exergy decomposition for the CRM configuration on the three grid levels. The residual in the exergy balance equation is important, but is reduced as the grid is refined. This is not associated to the accuracy of a particular term but a distributed contribution of $W \dot{\Gamma}$, the collective mechanical exergy deposition rate $\left(\dot{\mathcal{E}}_{m}\right)$ and total anergy generation $\left(\dot{\mathcal{A}}_{t o t}\right)$. For mechanical exergy, grid convergence is most likely tied to the reduction of numerical dissipation of the wing tip vortex on the finer grids (i.e. the term $\dot{\mathcal{E}}_{v w}$ ). As regards the rate of anergy generation, grid convergence is most likely tied to the more accurate calculation of primitive variable gradients (viscous dissipation, thermal conduction, shockwave). On all three grid levels, the absolute value of $W \dot{\Gamma}$ is within 1 count from that of the near-field drag coefficient.

As in the airfoil case, exergy analysis can be used to identify flow regions where loss of exergy (i.e. loss of work potential) is dominant either due to viscous dissipation or thermal mixing (see Fig. 8). On the same figure, a downstream extension of the control volume can portray the reduction of mechanical exergy components and increase of anergy generation due to irreversible processes within the volume.

\section{B. Quantification of spurious anergy generation}

The study of Section IV.A has demonstrated that numerical errors (inadequate convergence, numerical discretization) can lead to the exergy balance not being verified on the discrete level. The error in the verification of the exergy balance can be partly attributed to non-physical entropy generation. The objective of the present study is the detection and quantification of this spurious entropy, with the aim of limiting the dependence of an accurate exergy analysis to the mesh quality and numerical discretization. 

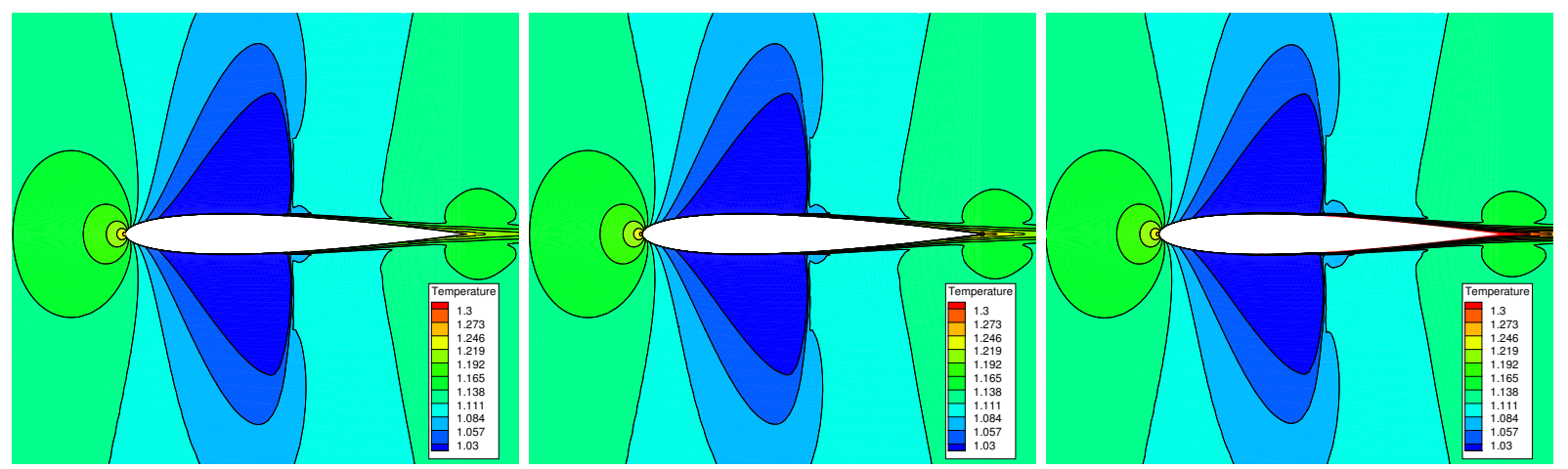

Fig. 9 Visualisation of the temperature field for the NACA0012 solutions at $M_{\infty}=0.8$ on the $512 \times 128$ grid with different wall boundary conditions: adiabatic (left), $T_{w}=350 \mathrm{~K}$ (middle) and $T_{w}=450 \mathrm{~K}$ (right).

A possibility would be the use of integration volumes detected by physical criteria associated to specific flow phenomena, rather than integrating in an enlarged volume which encloses the aerodynamic body (see Fig. 1). This strategy is similar to the quantification of spurious drag for far-field drag extraction methods [2, 16]. As such, integration volumes are based on the detection of three types of regions depending on the flow mechanisms contained therein: viscous, thermal and shockwaves. The term $\dot{\mathcal{A}}_{w}$ is already integrated in such a localized surface $\partial V_{w}$ (Eq. (10)).

In the present analysis, the rate of viscous anergy generation (term $\dot{\mathcal{A}}_{\Phi}$ ) is integrated in a reduced volume $\mathcal{V}_{\Phi}$, rather than the complete volume $\mathcal{V}_{o}$ (see Eq. (8)). For flows involving thermal transfer, the definition of a thermal integration volume based on physical criteria has also been developed. The term $\dot{\mathcal{A}}_{\nabla T}$ is then integrated in the volume $\mathcal{V}_{T}$, rather than the complete volume $\mathcal{V}_{o}$ (see Eq. $(9)$ ). Following the detection of these two volumes, the integration of $\dot{\mathcal{A}}_{\Phi}, \dot{\mathcal{A}}_{\nabla T}$ in $\mathcal{V}_{o} \backslash \mathcal{V}_{\Phi}$ and $\mathcal{V}_{o} \backslash \mathcal{V}_{T}$ respectively is used as a first measure of spurious anergy generation. Naturally, this strategy relies on an accurate definition of $\mathcal{V}_{\Phi}$ and $\mathcal{V}_{T}$.

The first case investigated is a NACA0012 airfoil in viscous flow. The reference conditions correspond to the viscous computations presented in Section IV.A Additional computations for a non-adiabatic wall boundary condition are also investigated, corresponding to a wall temperature of $T_{w}=350 \mathrm{~K}$ and $T_{w}=450 \mathrm{~K}$ (see Fig. 9). It is reminded that the term $\dot{\mathcal{E}}_{q}$ in the exergy balance is non-zero in these cases due to the non-adiabatic airfoil surface (see Eqs. (2), 5).

\begin{tabular}{|c|c|c|c|c|c|c|c|c|}
\hline \multirow{2}{*}{ Grid } & \multirow{2}{*}{ Term $\left(\times 10^{-4}\right)$} & \multirow{2}{*}{ Volume } & \multicolumn{2}{|c|}{ Adiabatic wall } & \multicolumn{2}{|c|}{$T_{w}=350 \mathrm{~K}$} & \multicolumn{2}{|c|}{$T_{w}=450 \mathrm{~K}$} \\
\hline & & & $x_{\text {tp }}=2.0$ & $x_{\mathrm{tp}}=15.0$ & $x_{\text {tp }}=2.0$ & $x_{\mathrm{tp}}=15.0$ & $x_{\text {tp }}=2.0$ & $x_{\mathrm{tp}}=15.0$ \\
\hline \multirow{4}{*}{$n i=256$} & \multirow{2}{*}{$\dot{\mathcal{A}}_{\Phi}$} & $v_{o}$ & 82.68 & 85.77 & 73.32 & 76.31 & 60.27 & 63.11 \\
\hline & & $\mathcal{V}_{\Phi}$ & 82.65 & 85.74 & 73.29 & 76.28 & 60.24 & 63.08 \\
\hline & \multirow{2}{*}{$\dot{\mathcal{A}}_{\nabla T}$} & $\mathcal{V}_{o}$ & 1.01 & 1.11 & 65.04 & 68.08 & 358.42 & 376.27 \\
\hline & & $\mathcal{V}_{T}$ & 1.01 & 1.11 & 65.04 & 68.08 & 358.40 & 376.25 \\
\hline \multirow{4}{*}{$n i=512$} & \multirow{2}{*}{$\dot{\mathcal{A}}_{\Phi}$} & $\mathcal{V}_{o}$ & 84.59 & 87.74 & 75.28 & 78.34 & 62.20 & 65.11 \\
\hline & & $\mathcal{V}_{\Phi}$ & 84.56 & 87.71 & 75.25 & 78.31 & 62.17 & 65.08 \\
\hline & \multirow{2}{*}{$\dot{\mathcal{A}}_{\nabla T}$} & $\mathcal{V}_{o}$ & 1.06 & 1.16 & 67.02 & 70.12 & 371.28 & 389.56 \\
\hline & & $\mathcal{V}_{T}$ & 1.06 & 1.16 & 67.02 & 70.11 & 371.27 & 389.55 \\
\hline \multirow{4}{*}{$n i=1024$} & \multirow{2}{*}{$\dot{\mathcal{A}}_{\Phi}$} & $V_{o}$ & 85.02 & 88.16 & 75.74 & 78.79 & 62.67 & 65.57 \\
\hline & & $\mathcal{V}_{\Phi}$ & 84.98 & 88.13 & 75.71 & 78.76 & 62.64 & 65.54 \\
\hline & \multirow{2}{*}{$\dot{\mathcal{A}}_{\nabla T}$} & $\mathcal{V}_{o}$ & 1.07 & 1.17 & 67.52 & 70.60 & 374.69 & 392.95 \\
\hline & & $\mathcal{V}_{T}$ & 1.07 & 1.17 & 67.51 & 70.60 & 374.69 & 392.95 \\
\hline
\end{tabular}

Table 5 Effect of reduction of integration volumes for the viscous and thermal rate of anergy generation based on physical criteria for the NACA0012 case at $M_{\infty}=0.3$.

Results from the integration in reduced volumes defined based on physical criteria are shown in Tables 5 , 6 for the subsonic and transonic viscous NACA0012 case. These show that the viscous and thermal rate of anergy generation can 


\begin{tabular}{|c|c|c|c|c|c|c|c|c|}
\hline \multirow{2}{*}{ Grid } & \multirow{2}{*}{ Term $\left(\times 10^{-4}\right)$} & \multirow{2}{*}{ Volume } & \multicolumn{2}{|c|}{ Adiabatic wall } & \multicolumn{2}{|c|}{$T_{w}=350 \mathrm{~K}$} & \multicolumn{2}{|c|}{$T_{w}=450 \mathrm{~K}$} \\
\hline & & & $x_{\text {tp }}=2.0$ & $x_{\mathrm{tp}}=15.0$ & $x_{\text {tp }}=2.0$ & $x_{\mathrm{tp}}=15.0$ & $x_{\text {tp }}=2.0$ & $x_{\mathrm{tp}}=15.0$ \\
\hline \multirow{4}{*}{$n i=256$} & \multirow{2}{*}{$\dot{\mathcal{A}}_{\Phi}$} & $\mathcal{V}_{o}$ & 79.93 & 83.02 & 76.59 & 79.68 & 66.90 & 70.04 \\
\hline & & $\mathcal{V}_{\Phi}$ & 79.91 & 83.00 & 76.57 & 79.67 & 66.88 & 70.03 \\
\hline & \multirow{2}{*}{$\dot{\mathcal{A}}_{\nabla T}$} & $v_{o}$ & 9.30 & 9.98 & 15.43 & 16.43 & 52.88 & 55.70 \\
\hline & & $\mathcal{V}_{T}$ & 9.30 & 9.97 & 15.43 & 16.43 & 52.88 & 55.70 \\
\hline \multirow{4}{*}{$n i=512$} & \multirow{2}{*}{$\dot{\mathcal{A}}_{\Phi}$} & $\mathcal{V}_{o}$ & 80.84 & 83.65 & 77.54 & 80.36 & 68.00 & 70.88 \\
\hline & & $\mathcal{V}_{\Phi}$ & 80.82 & 83.63 & 77.52 & 80.34 & 67.98 & 70.85 \\
\hline & \multirow{2}{*}{$\dot{\mathcal{A}}_{\nabla T}$} & $v_{o}$ & 9.52 & 10.13 & 15.87 & 16.79 & 54.46 & 57.15 \\
\hline & & $\mathcal{V}_{T}$ & 9.51 & 10.13 & 15.87 & 16.79 & 54.45 & 57.15 \\
\hline \multirow{4}{*}{$n i=1024$} & \multirow{2}{*}{$\dot{\mathcal{A}}_{\Phi}$} & $v_{o}$ & 80.84 & 83.54 & 77.56 & 80.27 & 68.09 & 70.84 \\
\hline & & $\mathcal{V}_{\Phi}$ & 80.81 & 83.51 & 77.53 & 80.24 & 68.06 & 70.81 \\
\hline & \multirow{2}{*}{$\dot{\mathcal{A}}_{\nabla T}$} & $v_{o}$ & 9.52 & 10.12 & 15.92 & 16.82 & 54.75 & 57.39 \\
\hline & & $\mathcal{V}_{T}$ & 9.51 & 10.11 & 15.92 & 16.81 & 54.74 & 57.38 \\
\hline
\end{tabular}

Table 6 Effect of reduction of integration volumes for the viscous and thermal rate of anergy generation based on physical criteria for the NACA0012 case at $M_{\infty}=0.8$.

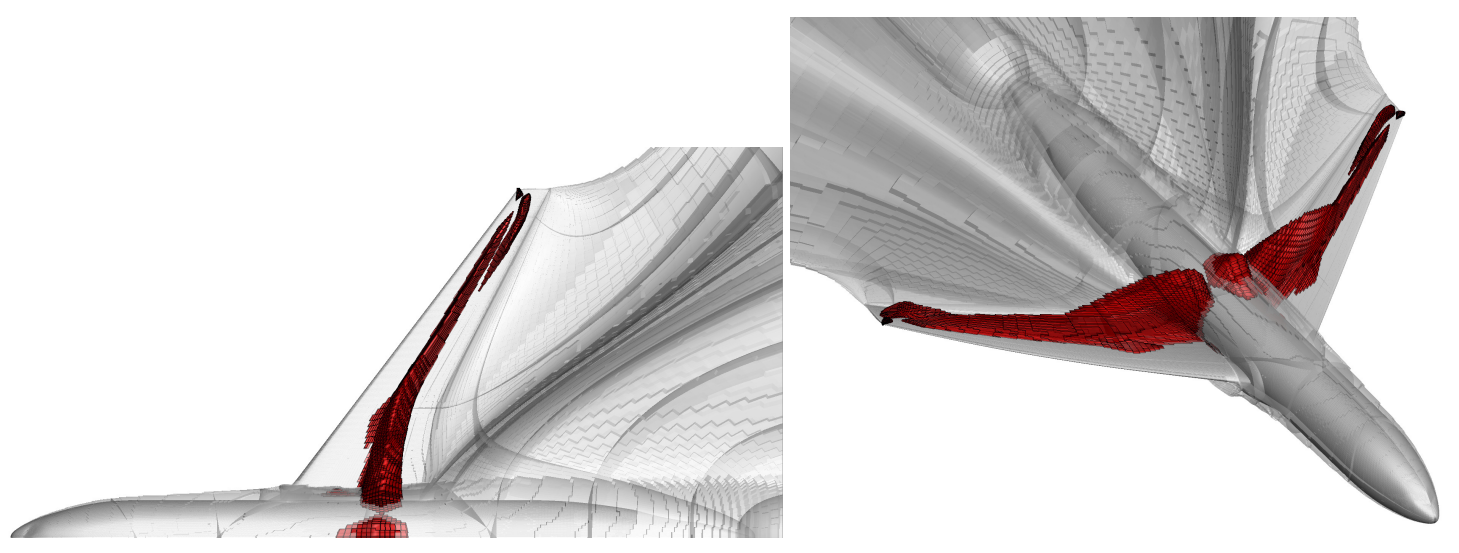

Fig. 10 Views of the integration volumes for $\dot{\mathcal{A}}_{\Phi}$ (grey) and $\dot{\mathcal{A}}_{w}$ (red) for the CRM case on the L3' grid. Only half of the CRM configuration was computed.

be integrated within the "physical" integration volumes, as long as the latter are appropriately defined so as to contain the viscous and thermal phenomena respectively. The value differences are lower than 0.05 counts for both $\dot{\mathcal{A}}_{\Phi}$ and $\dot{\mathcal{A}}_{\nabla T}$. Views of the three-dimensional integration volumes for the CRM case are shown in Fig. 10 Table 7 shows the values of $\dot{\mathcal{A}}_{\Phi}$ and $\dot{\mathcal{A}}_{\nabla T}$ integrated on the reduced volumes on the CRM case. As for the NACA0012 computations, the reduction of integration volumes results in small differences compared to the integration in the complete control volume. This further validates the precision of the physical detection criteria, but does not suffice to compensate for the exergy balance residual identified in the analysis of Section IV.A.

At a following step, spurious entropy production is directly calculated from the flow solution via the entropy flux. The rate of spurious anergy generation is defined as:

$$
\dot{\mathcal{A}}_{s p}=T_{\infty} \int_{\mathcal{V}_{s p}} \nabla \cdot(\rho \delta s \mathbf{V}) d \mathcal{V}
$$

The divergence of the entropy flux is shown in Fig. 11 for the NACA0012 airfoil in subsonic and transonic regime. Physical production of entropy is localized in boundary layers, the wake and shockwaves. In both regimes however, spurious entropy production is also observed close to the airfoil leading edge. This issue has been long identified by multiple authors and is also related to the production of spurious irreversible drag in this region [16]. On the transonic 


\begin{tabular}{cccccccc}
\hline \multirow{2}{*}{ Term $\left(\times 10^{-4}\right)$} & \multirow{2}{*}{ Volume } & \multicolumn{2}{c}{ L2 $^{\prime}$} & \multicolumn{2}{c}{ L3 $^{\prime}$} & \multicolumn{2}{c}{ L4 $^{\prime}$} \\
\cline { 2 - 8 } & & $x_{\mathrm{tp}}=90$ & $x_{\mathrm{tp}}=300$ & $x_{\mathrm{tp}}=90$ & $x_{\mathrm{tp}}=300$ & $x_{\mathrm{tp}}=90$ & $x_{\mathrm{tp}}=300$ \\
\hline \multirow{2}{*}{$\dot{\mathcal{A}}_{\Phi}$} & $\mathcal{V}_{o}$ & 122.72 & 126.04 & 125.86 & 129.40 & 128.89 & 132.59 \\
& $\mathcal{V}_{\Phi}$ & 122.70 & 126.02 & 125.84 & 129.38 & 128.87 & 132.57 \\
\cline { 2 - 8 }$\dot{\mathcal{A}}_{\nabla T}$ & $\mathcal{V}_{O}$ & 11.96 & 12.65 & 12.51 & 13.24 & 13.03 & 13.80 \\
& $\mathcal{V}_{T}$ & 11.96 & 12.64 & 12.50 & 13.24 & 13.02 & 13.79 \\
\hline
\end{tabular}

Table 7 Effect of reduction of integration volumes for the viscous and thermal rate of anergy generation based on physical criteria for the CRM case.

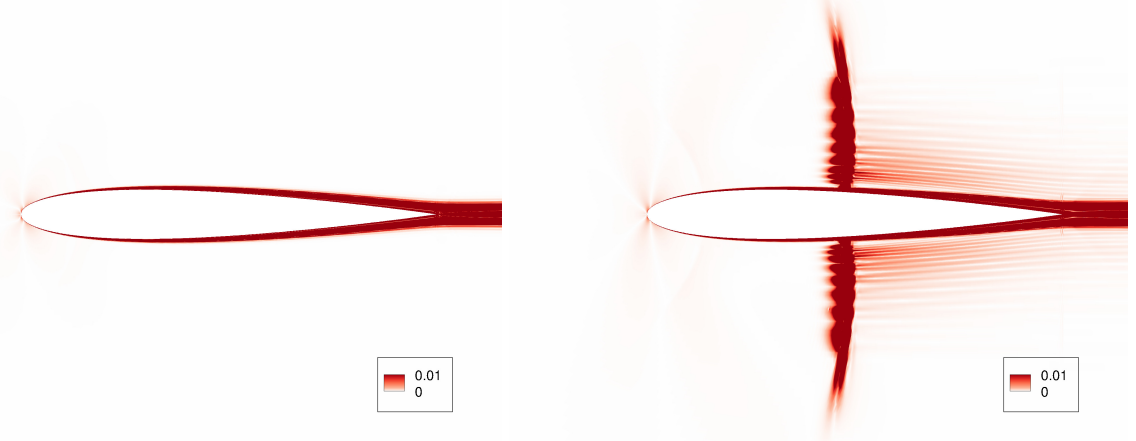

Fig. 11 Contour of the entropy flux divergence modulus around the NACA0012 airfoil at $M_{\infty}=0.3$ (left) and $M_{\infty}=0.8$ (right).



Fig. 12 Comparison of the residual of the exergy balance equation against the calculated rate of spurious anergy generation $\dot{\mathcal{A}}_{s p}$ and the reduction of integration volumes for $\dot{\mathcal{A}}_{\Phi}, \dot{\mathcal{A}}_{\nabla T}$. Values displayed in counts $\left(\times 10^{-4}\right)$.

case, spurious entropy production is also observed downstream of the shockwave, which is purely related to numerical discretization error.

To quantify the production of spurious entropy in the control volume, the integration volume for $\dot{\mathcal{A}}_{s p}$ is defined as $\mathcal{V}_{s p}=\mathcal{V}_{o} \backslash\left(\mathcal{V}_{\Phi} \cup \mathcal{V}_{T} \cup \mathcal{V}_{w}\right)$. This accounts for the loss of exergy through irreversible processes that are not associated to any of the physical integration volumes, and are thus considered to be of numerical origin.

A comparison between $\dot{\mathcal{A}}_{s p}$ and the residual of the discrete exergy balance equation (Eq. (2)) is shown in Fig. 12 for different cases. Results show that $\dot{\mathcal{A}}_{s p}$ corresponds well to the exergy balance residual in the inviscid airfoil cases, 
both in subsonic and transonic regime. On RANS airfoil calculations, several observations can be made. First, on the subsonic case with adiabatic wall, the exergy balance residual is well-balanced by $\dot{\mathcal{A}}_{s p}$. The exergy balance residual itself is however augmented on cases with non-adiabatic walls. This can be related to the creation of stronger thermal gradients in the airfoil wake that are not accurately captured on coarser grids. As regards RANS transonic cases, $\dot{\mathcal{A}}_{s p}$ accounts for only a part of the residual with the difference being even more important on the CRM case.

\section{Conclusions}

Several numerical investigations have been performed regarding the sensitivity of exergy analysis to numerical errors, specifically to the level of convergence and the grid density. The term $W \dot{\Gamma}$, associated to the net streamwise force acting on the aircraft, has been found to be sensitive at earlier stages of convergence. No similar sensitivity has been identified for the rest of the terms of the exergy balance. Furthermore, it was shown that the exergy balance equation may not be satisfied at the discrete level due to numerical approximation errors inherent in numerical computations. The residual of this balance was found to reduce with refinement of the grid, as physical phenomena represented by the exergy formulation are more accurately captured. In an attempt to balance this residual, a strategy has been presented for the identification and quantification of spurious anergy generation (i.e. loss of work potential due to irreversible processes). This is based on the definition of integration volumes based on physical criteria. The resulting term has been found to at least partly compensate the residual in the discrete exergy balance equation. Nonetheless, it does not suffice to balance the exergy balance residual, suggesting that the latter can be the result of a more complex interaction of physical and numerical effects. Further refinements of this strategy thus appear interesting, since exergy analysis itself remains a powerful and promising tool for aerodynamic design and performance assessment.

\section{Acknowledgments}

The present research work was partially funded by the French Directorate General for Civil Aviation (DGAC) through the SUBLIME convention. The studies presented have used the elsA software, whose development is partially funded by its three co-owners: ONERA, Airbus, SAFRAN.

\section{References}

[1] Van Der Vooren, J., and Destarac, D., "Drag/thrust analysis of jet-propelled transonic transport aircraft; definition of physical drag components," Aerospace Science and Technology, Vol. 8, No. 6, 2004, pp. 545-556.

[2] Destarac, D., Far-Field / Near-Field Drag Balance and Applications of Drag Extraction in CFD, In: CFD-based Aircraft Drag Prediction and Reduction, VKI Lecture Series 2003, Von Karman Institute for Fluid Dynamics, Rhode Saint Genèse, February 3-7, 2003, National Institute of Aerospace, Hampton (VA), November 3-7, 2003.

[3] Uranga, A., Drela, M., Greitzer, E. M., Hall, D. K., Titchener, N. A., Lieu, M. K., Siu, N. M., Casses, C., Huang, A. C., Gatlin, G. M., et al., "Boundary Layer Ingestion Benefit of the D8 Transport Aircraft," AIAA Journal, Vol. 55, No. 11, 2017, pp. 3693-3708.

[4] Wiart, L., Atinault, O., Boniface, J.-C., and Barrier, R., "Aeropropulsive performance analysis of the NOVA configurations," 30th Congress of the International Council of the Aeronautical Sciences (ICAS), Daejeon, Korea, September 2016.

[5] Drela, M., "Power balance in aerodynamic flows," AIAA Journal, Vol. 47, No. 7, 2009, pp. 1761-1771.

[6] Arntz, A., "Civil Aircraft Aero-thermo-propulsive Performance Assessment by an Exergy Analysis of High-fidelity CFD-RANS Flow Solutions,” Ph.D. thesis, Lille 1 Université - Sciences et Technologies, Lille, France, 2014.

[7] Arntz, A., Atinault, O., and Merlen, A., "Exergy-Based Formulation for Aircraft Aeropropulsive Performance Assessment: Theoretical Development,” AIAA Journal, Vol. 53, No. 6, 2015, pp. 1627-1639.

[8] Arntz, A., and Hue, D., "Exergy-Based Performance Assessment of the NASA Common Research Model," AIAA Journal, Vol. 54, No. 1, 2016, pp. 88-100.

[9] Couilleaux, A., and Arntz, A., "Exergy analysis for a CFD-based turbofan exhaust mixer performance improvement," 53rd $3 A F$ International Conference on Applied Aerodynamics, Salon de Provence, France, 26-28 March, 2018.

[10] Wiart, L., and Negulescu, C., "Exploration of the Airbus "NAUTILIUS" engine integration concept," 31st Congress of the International Council of the Aeronautical Sciences (ICAS), Belo Horizonte, Brazil, September 2018. 
[11] Benoit, C., Péron, S., and Landier, S., "Cassiopee: a CFD pre-and post-processing tool," Aerospace Science and Technology, Vol. 45, 2015, pp. 272-283.

[12] Rumsey, C., Wedan, B., Hauser, T., and Poinot, M., "Recent updates to the CFD general notation system (CGNS)," 50th AIAA Aerospace Sciences Meeting including the New Horizons Forum and Aerospace Exposition, AIAA Paper 2012-1264, American Institute of Aeronautics and Astronautics, 2012.

[13] Tailliez, C., and Arntz, A., "CFD assessment of the use of exergy analysis for losses identification in turbomachines flows," 53rd 3AF International Conference on Applied Aerodynamics, Salon de Provence, France, 26-28 March, 2018.

[14] Cambier, L., Heib, S., and Plot, S., "The Onera elsA CFD software: input from research and feedback from industry," Mechanics \& Industry, Vol. 14, No. 3, 2013, pp. 159-174.

[15] Vassberg, J. C., and Jameson, A., "In pursuit of grid convergence for two-dimensional Euler solutions," Journal of Aircraft, Vol. 47, No. 4, 2010, pp. 1152-1166.

[16] Destarac, D., “Three-Component Breakdown of Spurious Drag in Computational Fluid Dynamics,” Journal of Aircraft, Vol. 52, No. 4, 2015, pp. 1336-1344. doi:10.2514/1.C032924.

[17] Vassberg, J. C., "A Unified Baseline Grid About the Common Research Model Wing/Body for the Fifth AIAA CFD Drag Prediction Workshop," 29th AIAA Applied Aerodynamics Conference, American Institute of Aeronautics and Astronautics, 2011.

[18] Hue, D., "Fifth Drag-Prediction Workshop: ONERA Investigations with Experimental Wing Twist and Laminarity," Journal of Aircraft, Vol. 51, No. 4, 2014, pp. 1311-1322. 